\title{
474686 - PERIOPERATIVE MORBIDITY AND CORONARY REVASCULARAZATION SURGERY
}

\author{
Ronit Lavi, MD ${ }^{1}$, Jacek Karski, MD, FRCPC ${ }^{1}$, Robert James Cusimano, MD ${ }^{2}$, Jo \\ Carroll, $\mathbf{R N}^{1}$, George Djaiani, $\mathbf{M D}^{1}$ \\ 1. Anesthesia, Toronto General Hospital, Toronto, ON, Canada \\ 2. Cardiac Surgery, Toronto General Hospital, Toronto, ON, Canada
}

Introduction: Coronary artery bypass graft (CABG) surgery is performed either with or without cardiopulmonary bypass (CPB). The objective of this study was to determine perioperative morbidity with the conventional on-pump, off-pump, and off-pump converted to on-pump CABG surgery.

Methods: After REB approval we examined data collected prospectively on patients undergoing primary elective CABG surgery as part of an Anesthesia Perioperative Outcomes Database. Based on the type of CABG surgery all patients were classed into three groups: conventional on-pump, off-pump, and off-pump converted to on-pump. Patients were matched for age, sex, and preoperative risk factors. Perioperative parameters, and postoperative complications were evaluated using univariate analysis. Results: A total of 330 patients were studied. Anesthesia and surgical management were standardized. The reasons for conversion included intra myocardial left anterior descending artery and hemodynamic instability. The number of grafts performed in the Off pump group was lower compared to CPB and Off pump converted groups; $2.8 \pm 0.9$, $3.6 \pm 1 ; 3.4 \pm 1, p=0.001$, respectively. Blood product transfusion rates, duration of intubation, prevalence of atrial fibrillation, and length of hospital stay are presented in the table.

Discussion: Blood product transfusion rates and hospital length of stay were increased in the Off pump converted group. Postoperative atrial fibrillation was more frequent in the On-pump group. The present study demonstrates that intraoperative conversion from elective off-pump surgery to on-pump surgery is associated with higher blood product transfusion rates and longer hospital length of stay.

References: None 
Table 1

\begin{tabular}{|l|l|l|l|l|}
\hline & Off Pump & Converted & On-pump & P-value \\
\hline AGE years & $62 \pm 10$ & $63 \pm 10$ & $64 \pm 4$ & 0.1 \\
\hline SEX male (\%) & $84(76)$ & $88(80)$ & $94(85)$ & 0.2 \\
\hline MI n(\%) & $50(45)$ & $50(45)$ & $39(35)$ & 0.2 \\
\hline DM n(\%) & $33(30)$ & $38(34)$ & $42(38)$ & 0.4 \\
\hline HTN n(\%) & $56(50)$ & $65(60)$ & $73(66)$ & 0.09 \\
\hline CHF n(\%) & $8(7)$ & $6(5)$ & $2(2)$ & 0.1 \\
\hline PVD n(\%) & $8(7)$ & $13(12)$ & $6(5)$ & 0.2 \\
\hline$\%$ Change HB & $29 \pm 11$ & $28 \pm 10$ & $26 \pm 8.6$ & 0.1 \\
\hline$\%$ Change Cr & $7.3 \pm 73$ & $6.4 \pm 32$ & $-0.8 \pm 26$ & 0.4 \\
\hline Number of Grafts & $2.8 \pm 0.9$ & $3.4 \pm 1$ & $3.6 \pm 1$ & 0.001 \\
\hline Pump Run (minutes) & & $76 \pm 24$ & $92 \pm 24$ & 0.0001 \\
\hline X clamp (minutes) & & $59 \pm 18$ & $71 \pm 20$ & 0.0001 \\
\hline Peri PRBC & $0.20 \pm 0.5$ & $1.3 \pm 1.6$ & $0.3 \pm 0.8$ & 0.001 \\
\hline Peri FFP & $0.09 \pm 0.5$ & $0.40 \pm 1.2$ & $0.1 \pm 0.5$ & 0.0032 \\
\hline Peri Plt & $0.09 \pm 0.7$ & $0.7 \pm 2.3$ & $0.04 \pm 0.2$ & 0.0009 \\
\hline Intubation (hours) & $7.3 \pm 4$ & $13 \pm 19$ & $7 \pm 5$ & 0.0007 \\
\hline Length of stay : Days & $7.4 \pm 4.2$ & $9.4 \pm 7.3$ & $6.7 \pm 2.3$ & 0.0001 \\
\hline Post AFIB (\%) & $14(12.7)$ & $22(20)$ & $37(33)$ & 0.04 \\
\hline
\end{tabular}

MI: myocardial infarction; DM: diabetes mellitus; HTN: hypertension; CHF: congestive heart failure; PVD: peripheral vascular disease; \% Change $\mathrm{Hb}$ : the perioperative change in Hemoglobin levels; \% Change $\mathrm{Cr}$ : the perioperative change in creatinine: X clamp: cross calmp time; Peri PRBC: perioperative red blood cells administered; Peri FFP: perioperative fresh frozen plasma administered; Peri Plt: perioperative platelets administered; Post AFIB: the occurrence of new onset perioperative atrial fibrillation 